\title{
EchoGéo
}

$58 \mid 2021$

Varia

\section{Les transects photographiques : à la recherche des discontinuités dans le paysage}

\section{Morgane Flégeau}

\section{OpenEdition}

1 Journals

Édition électronique

URL : https://journals.openedition.org/echogeo/22641

DOI : 10.4000/echogeo.22641

ISSN : 1963-1197

Éditeur

Pôle de recherche pour l'organisation et la diffusion de l'information géographique (CNRS UMR 8586)

Référence électronique

Morgane Flégeau, « Les transects photographiques : à la recherche des discontinuités dans le paysage », EchoGéo [En ligne], 58 | 2021, mis en ligne le 31 décembre 2021, consulté le 12 février 2022 URL : http://journals.openedition.org/echogeo/22641 ; DOI : https://doi.org/10.4000/echogeo.22641

Ce document a été généré automatiquement le 12 février 2022.

EchoGéo est mis à disposition selon les termes de la licence Creative Commons Attribution - Pas d'Utilisation Commerciale - Pas de Modification 4.0 International (CC BY-NC-ND) 


\title{
Les transects photographiques : à la recherche des discontinuités dans le paysage
}

\author{
Morgane Flégeau
}

\section{Introduction}

1 La photographie est aujourd'hui largement plébiscitée comme un outil de production de données pour les recherches en sciences humaines et sociales, notamment en géographie. En particulier, l'étude des paysages dans cette discipline s'est adjoint depuis longtemps déjà la photographie comme un outil d'analyse (Métailié, 1995). La mobilisation de cet outil dans les travaux de recherche nécessite une réflexion sur la méthodologie spécifique qu'elle implique. Cet article présente la mise en œuvre d'une méthode exploratoire mobilisant des transects photographiques pour approcher les logiques d'organisation dans le paysage. Une base de données photographique a été constituée, selon un protocole de production des clichés spécifique. Mobilisée dans le cadre d'un travail de thèse de doctorat, la photographie permet de décrire les " arrangements paysagers» composant l'espace de la Ceinture verte d'île-de-France correspondant à des types d'interface entre plusieurs modes d'occupation du sol, que l'espace soit bâti - sous toutes ses formes - ou non-bâti. Proposé en 1983 par la Région Île-de-France, cet outil d'aménagement a été placé volontairement à cheval entre l'agglomération dense et la couronne périurbaine, sur un périmètre allant de 10 à 30 kilomètres du centre de Paris, constitué de 436 communes. La définition de l'appartenance des communes au périmètre de la Ceinture verte a été fixé par un ratio de $60 \%$ d'espaces ouverts, boisés, agricoles et naturels et de $40 \%$ d'espaces urbains. Les ceintures vertes, d'abord conçues en Grande Bretagne, ont été ensuite une constante de l'idéologie de la planification des villes, afin d'en limiter l'extension, en Europe et dans le monde, depuis le début XXe siècle (Amati, 2008). Dans le cas francilien, l'application de cet outil a cependant été très limitée, faute notamment de véritables contraintes réglementaires (Laruelle et Legenne, 2004a). La Ceinture verte d'Île-de-France a ainsi 
largement échappé à une planification d'ensemble. Les paysages inclus dans ce périmètre, aux lisières de la métropole parisienne, sont aujourd'hui le lieu d'un enchevêtrement d'espaces agricoles, de lotissements, de zones d'activités, de centres villes anciens, de boisements ou encore de friches et de délaissés, et s'organisent dans une apparente cacophonie. Malgré ce relatif échec, réapparait aujourd'hui l'idée d'une ceinture verte dans différents cercles de l'action publique. Cette mise en avant va de pair avec la nécessité revendiquée de préserver les espaces végétalisés des marges des métropoles. Acteurs de l'aménagement et habitants attendent ainsi des espaces libres d'urbanisation des fonctions paysagères et de loisirs (Poulot, 2013), de ressources environnementales et écologiques (Arrif et al., 2011) ou encore de santé publique (Bolon et al., 2019).

2 Notre travail de thèse proposait une réflexion sur la manière dont les caractéristiques de la Ceinture verte - et notamment l'emprise spatiale de ses espaces végétalisés participent à la constitution de territoires originaux, répondant aux attentes environnementales des habitants et d'acteurs des politiques publiques d'aménagement en quête d'une métropole accueillant davantage de nature (Flégeau, 2018a). Afin d'appréhender les reconfigurations spatiales et paysagères au sein de cet espace, un raisonnement multiscalaire a été mobilisé, en faisant appel en particulier à deux outils d'analyse, l'un cartographique et l'autre photographique. Le média photographique permet d'enrichir la réflexion sur les agencements paysagers en Ceinture verte dans un espace largement modifié depuis les années 1970 et 1980 . Une méthode exploratoire a mobilisé des transects photographiques, en s'appuyant sur l'identification de huit faisceaux de 20 kilomètres chacun et comprenant une série de clichés photographiques pris tous les kilomètres, au cours de deux campagnes réalisées aux mois de juin des années 2014 et 2015. Une base de données de plus de 800 photographies a ainsi été constituée. Nous souhaitions identifier l'agencement du paysage et vérifier les possibilités d'organisation spatiale en Ceinture verte mais aussi mettre en regard les notions de discontinuités spatiales (Brunet et al., 1997) et d'appropriation par les acteurs et habitants du territoire par le biais d'un outil visuel. Cette enquête photographique fut aussi l'occasion d'un parcours au sol et de l'expérimentation sur le terrain des zones de transition ou de ruptures repérables sur les cartes le long d'axes ne suivant pas les axes de communication. Enfin, ce travail a permis une amorce de réflexion sur les spécificités de l'espace public dans le périurbain proche de Paris. Nous proposerons dans un premier temps de situer la méthode de la photographie comme outil d'analyse paysagère, complémentaire de l'outil cartographique, dans une démarche géographique. La méthode ainsi que le protocole construit seront ensuite présentés. Nous mettrons enfin en avant l'apport de la photographie pour la compréhension des discontinuités et de l'appropriation de l'espace aux marges de la métropole parisienne.

\section{Qualifier les paysages de la Ceinture verte par un échantillonnage photographique systématique}

3 La photographie est ici utilisée comme outil d'analyse du paysage après un premier travail cartographique. Ce dernier avait permis de proposer une première qualification de l'espace de Ceinture verte, celui d'un espace périurbain hétérogène. La photographie 
permet, elle, de décrypter à l'échelle fine les agencements, les effets de rupture et les transitions paysagères repérés au préalable.

\section{Deux approches complémentaires : la cartographie et la photographie}

4 L'étude de l'organisation spatiale de la Ceinture verte à l'échelle régionale avait ainsi été réalisée au travers d'une analyse cartographique. Celle-ci avait d'abord permis de définir des types de configurations paysagères et des modalités de contact entre zones urbanisées et espaces libres d'urbanisation. Elle avait aussi été l'occasion, dans une perspective historique et dynamique, d'appréhender le rapport entre la planification et le développement non maitrisé de l'urbain. Ce travail s'est fondé sur l'utilisation de la carte topographique au $100000^{\text {e }}$ proposée en 2011 par l'Institut géographique national (IGN), et retravaillée ensuite à l'aide d'un Système d'Information géographique (SIG) afin d'obtenir une nomenclature en cinq classes d'occupation du sol: le bâti, l'espace agricole, l'espace boisé, les cours d'eau ainsi que les zones humides. Une série de cartes diachroniques a été réalisée à l'échelle régionale. Ces cartes représentent l'évolution de l'occupation du sol depuis 1978 et reviennent sur la progression de la tache urbaine de Paris au cours des cinquante dernières années. Cette classification est simplifiée. Il aurait pu être intéressant de mobiliser les classifications réalisées par l'IAU (Institut d'aménagement et d'urbanisme d'île-de-France) au travers de l'outil du Mode d'Occupation du Sol. Toutefois, ses premières productions datent de 1982. Les cartes topographiques permettent, elles, d'avoir une profondeur historique permettant d'appréhender les transformations spatiales à une échelle régionale. Cette cartographie a permis de mettre en œuvre des traitements qualitatifs et notamment de spatialiser les espaces non bâtis les plus vulnérables au processus d'urbanisation et d'identifier les phénomènes de mitage et d'enclavement des espaces végétalisés en Ceinture verte. Nous avons ainsi identifié des grands types d'arrangements paysagers. Dans un espace largement transformé depuis que le phénomène de périurbanisation et de construction des villes nouvelles s'est amorcé dans les années 1970 et 1980, l'utilisation, en complément, d'une méthode photographique permet d'affiner le propos sur les dynamiques spatiales à l'œuvre.

\section{L'outil photographique : changement d'échelle et de point de vue spatial}

L'approche cartographique est particulièrement utile pour décrire des évolutions dans le temps et des types d'organisation de l'espace dans l'ensemble de la Ceinture verte. La mise en œuvre d'une méthode fondée sur la photographie induit, elle, un changement d'échelle, faisant passer de l'échelon régional au local. L'usage de la photographie permet d'analyser de manière fine l'occupation du sol le long de chaque tracé proposé et de de restituer les continuités et les discontinuités du paysage francilien observées au préalable grâce à la cartographie. L'analyse scientifique repose sur l'identification et la description de chaque plan capturé par les photographies en vue de passer à l'interprétation. Par ailleurs, l'outil photographique est utilisé comme un moyen de collecter et de stocker dans une base de données des "traces iconographiques" (Meyer et Papinot, 2017) des paysages observés à l'œil en Ceinture verte. Nous 
constituons de cette manière notre propre fond photographique visant à renseigner l'espace d'étude.

6 La photographie conduit aussi à un changement de cadre de la spatialité. Le travail de décryptage des paysages se fait au sol. Le regard de l'observateur se rapproche alors considérablement de son terrain d'étude. Le protocole de recherche que nous avons suivi visait à une production d'images en grand nombre, réalisées selon des critères précis à intervalles réguliers et le long d'axes radiaux. Le nombre de photographies collectées implique ainsi une analyse sérielle qui induit un changement de point de vue (Gendrot 2002), l'analyse de chaque photographie étant replacée dans celle de l'ensemble des clichés pris le long du transect. La méthode est ici en quelque sorte radicalisée, la photographie permettant de saisir les objets et les formes constituant le paysage dans un rapport d'immédiateté.

7 Au-delà de la description fine de l'occupation du sol, l'usage de la photographie permet de tirer un ensemble de considérations d'une grande richesse, qui ne sont pas perceptibles par l'approche de la cartographie de l'occupation des sols, sur la topographie, les composantes végétales ou bien architecturales et, ce qui est du domaine du sensible, les ambiances et les textures. Ces considérations font intervenir un rapport subjectif, induit par la prise de clichés photographiques, avec l'objet d'étude. L'appréhension de la dimension sensible des lieux est le premier enjeu de subjectivité soulevé dans ce travail. Le second interroge le rapport entre l'observateur/ enquêteur à son terrain puisque nous avons eu recours à ce travail dans une optique de faire l'expérience personnelle et de connaitre notre espace d'étude, celui de la Ceinture verte d'île-de-France. Il s'agissait donc de construire notre propre subjectivité. Comme le rappelle A. Marshall (2009), la subjectivité du géographe peut donner lieu à une " interprétation et à une analyse scientifique de la photographie », dans des conditions où la méthode est appliquée avec rigueur. Dans notre cas, il s'agissait d'inscrire l'analyse photographique dans une métrique et une progression. Cette subjectivité est intégrée dans notre analyse, en mettant en avant les choix opérés et est mise en perceptive avec un travail sur l'espace vécu par les habitants, thématique au cœur du travail de thèse.

\section{Un media pour appréhender les diverses appropriations de l'espace}

8 La méthode des transects photographiques a une place de pivot dans la méthodologie déployée dans le travail de thèse. En effet, elle s'inscrit à la fois en complément de la cartographie mais également en amont d'une enquête qualitative par entretiens semidirectifs auprès d'acteurs et habitants dans trois terrains d'étude en Ceinture verte. Dans l'espace périurbain, ces modes d'appropriation sont variés et servent à la construction de territoires singuliers (Poulot et al., 2016). L'utilisation de l'outil photographique permet ainsi de décrire un paysage à l'échelle où il est perçu par les habitants, de déceler également les traces d'appropriation visibles dans le paysage et de fournir des indices sur les valeurs et les fonctions visibles dans le paysage, attribuées aux différents espaces. La constitution d'un fonds photographique apporte de nombreuses informations sur l'appropriation des espaces de nature en Ceinture verte par les habitants et leurs aménagements par les pouvoirs publics. Les campagnes de prises de vue ont été réalisées au début du travail de thèse. La photographie permet de se forger une véritable connaissance du territoire par l'expérience de terrain. Cette 
pratique du terrain réalisée grâce aux photographies doit pouvoir être comparée par la suite avec celle des personnes qui résident dans ce territoire. Le media photographique prend donc une place particulière dans la relation du chercheur avec son terrain. La spatialité de la Ceinture verte est perçue au travers de la photographie. L'appréhension de l'appropriation des espaces par ses habitants se fait via le repérage des traces dans l'espace.

de compléter cette étude, une enquête pour appréhender les représentations et valeurs paysagères attribuées par les habitants a également été réalisée, avec l'aide d'une planche photographique. L'utilisation de photographies en appui d'entretiens semi-directifs a largement été mobilisée par des géographes dans le sillage d'Y. Luginbühl (1989) afin de faciliter la compréhension des représentations du paysage et permet ainsi de faire appel à la subjectivité de chacun (Lelli et Paradis-Mandive, 2000 ; Lelli, 2003 ; Bigando, 2013).

\section{La méthode des coupes paysagères : un mode d'analyse exploratoire de l'agencement spatial des paysages}

10 À l'échelle régionale, l'analyse cartographique de l'occupation du sol a montré les logiques différenciées d'urbanisation ainsi qu'une grande hétérogénéité de l'espace de Ceinture verte (Flégeau, 2018a). Pour approfondir l'agencement des paysages, l'étude de plusieurs segments radiaux en Ceinture verte permet de cerner les dynamiques territoriales et de souligner des tendances, des ruptures et des divergences. On cherche à y déceler des modèles d'organisation paysagère.

11 La réalisation des coupes paysagères a suivi un protocole précis. Pour décrire les paysages de la Ceinture verte, des transects sont tracés, traversant son épaisseur. Les transects sont couramment employés en géographie, notamment en biogéographie (Alexandre, 1994 ; Lizet et de Ravignan, 1994). Dans cette logique, sont étudiées les communautés biologiques en traçant une ligne-échantillon orientée par un gradient, le long de laquelle des observations et des mesures sont réalisées. Dans cette méthode, on reprend la logique du gradient pour étudier les évolutions du paysage le long de cette ligne échantillon, que l'on a appelée transect ou coupe paysagère. Tracer des segments radiaux permet de traverser l'épaisseur de la Ceinture verte, en adoptant la logique concentrique de cette entité et d'interroger le gradient d'urbanisation. Ce gradient d'urbanisation est-il continu? Les logiques d'urbanisation sont-elles pour l'essentiel radioconcentriques? La sélection de des segments radiaux pertinents pour placer nos transects s'est faite à partir d'observations faites au travers de l'analyse cartographique, notamment grâce à l'analyse diachronique de l'occupation du sol et à l'analyse spatialisée des caractéristiques socio-économiques à l'échelle de l'ensemble de la Ceinture verte. Ces critères de vérification de logiques spatiales étaient croisés avec la présence de projets d'aménagement et des périmètres de protection des espaces non bâtis, comme ceux des parcs naturels régionaux.

Huit transects sont ainsi définis, comprenant chacun vingt kilomètres, permettant de couvrir l'ensemble de l'épaisseur de la Ceinture verte, allant de sa frange interne à sa frange externe, suivant le périmètre établi par la Région Île-de-France. Le long de ces axes, un point d'observation est fixé à chaque kilomètre. Cette distance métrique a été 
choisie afin d'observer le paysage à une échelle fine et de l'articuler avec l'échelon de la cartographie régionale, moins précis, en identifiant les arrangements paysagers. L'intervalle d'un kilomètre permet d'acquérir une certaine précision d'observation et de noter les évolutions paysagères de manière rigoureuse. À chaque point rencontré, une ou plusieurs photographies sont prises dans les quatre directions cardinales. Pour cet exercice, il est nécessaire, autant que faire se peut, de conserver le même cadrage, la même hauteur - à hauteur d'œil -, en prenant en compte les bases de la technique photographique. Pour chacun des arrêts, entre quatre et six clichés photographiques ont été pris. La focale des photographies est standard. En outre, les coordonnées GPS de chaque photographie sont renseignées afin d'être intégrées dans un système d'information géographique.

Illustration 1 - Localisation des transects paysagers réalisés dans le périmètre de la Ceinture verte en juin 2014 et 2015

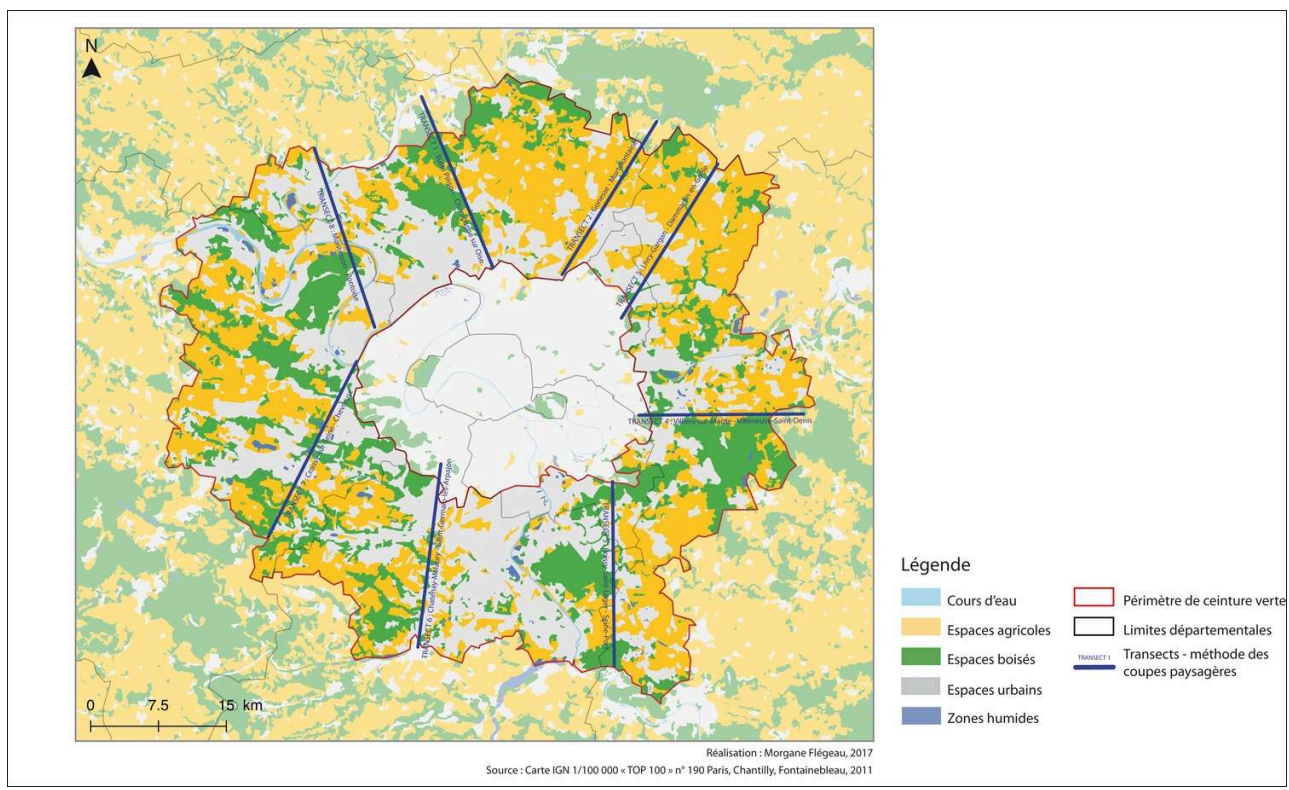

Les deux campagnes photographiques ont été menées à la même période de l'année afin de respecter la saison, en particulier pour que le couvert végétal présente un aspect similaire. En revanche, l'obtention d'un même éclairage lors de chaque séance est plus complexe, les conditions météorologiques étant généralement favorables, mais pas toujours garanties. Enfin, il était parfois impossible d'accéder à certains points car l'espace concerné y était privé et clôturé. Lorsque cela était possible, le point a été décalé de quelques mètres. Dans le cas inverse, l'impossibilité d'accès au point était notée. Au total, 15 points n'ont pas pu être photographiés. Dans un souci de continuité, nous avons complété l'information - lorsqu'elle manquait - par des photographies satellites. Ces dernières ne remplacent pas l'interprétation paysagère mais elles sont utiles afin de se faire une idée de l'évolution du paysage sur l'ensemble des transects. Cette information constitue par ailleurs un résultat pertinent pour décrypter la fragmentation paysagère ainsi que l'appropriation qui est faite de l'espace.

Le traitement qualitatif de la base de données a permis de donner «corps » à chacun des transects, en décryptant une organisation spatiale sur le mode continu-discontinu. Les types d'occupation du sol sur chaque transect sont analysés selon la distance à la frange interne de Ceinture verte. Pour formaliser les informations collectées, une 
interprétation est réalisée à partir des observations paysagères sur les photographies prises en chaque point du transect. Ce travail a conduit à l'élaboration d'une typologie des paysages rencontrés le long des transects. Une analyse des différents types de ruptures paysagères et des modes d'appropriation de l'espace photographié a ensuite été proposée.

\section{Une lecture des paysages de la Ceinture verte par la photographie}

\section{Les arrangements paysagers : des logiques géographiques visibles dans le paysage}

L'analyse paysagère montre à première vue un relatif « désordre » dans l'organisation des paysages dans l'espace couvert par les photographies. L'espace traversé apparaît comme une mosaïque, alternant espaces ouverts et espaces urbains (illustration 2). Le relatif équilibre entre espaces non bâtis et zones urbanisées, qui constitue selon les instances régionales l'originalité du périmètre de Ceinture verte, ne se vérifie pas dans l'analyse photographique conduite dans ce travail. Une forte dominance de l'artificialisation des paysages, notamment urbaine, se révèle dans notre travail photographique.

Illustration 2 - Occupation du sol principale à chaque point photographié le long des transects

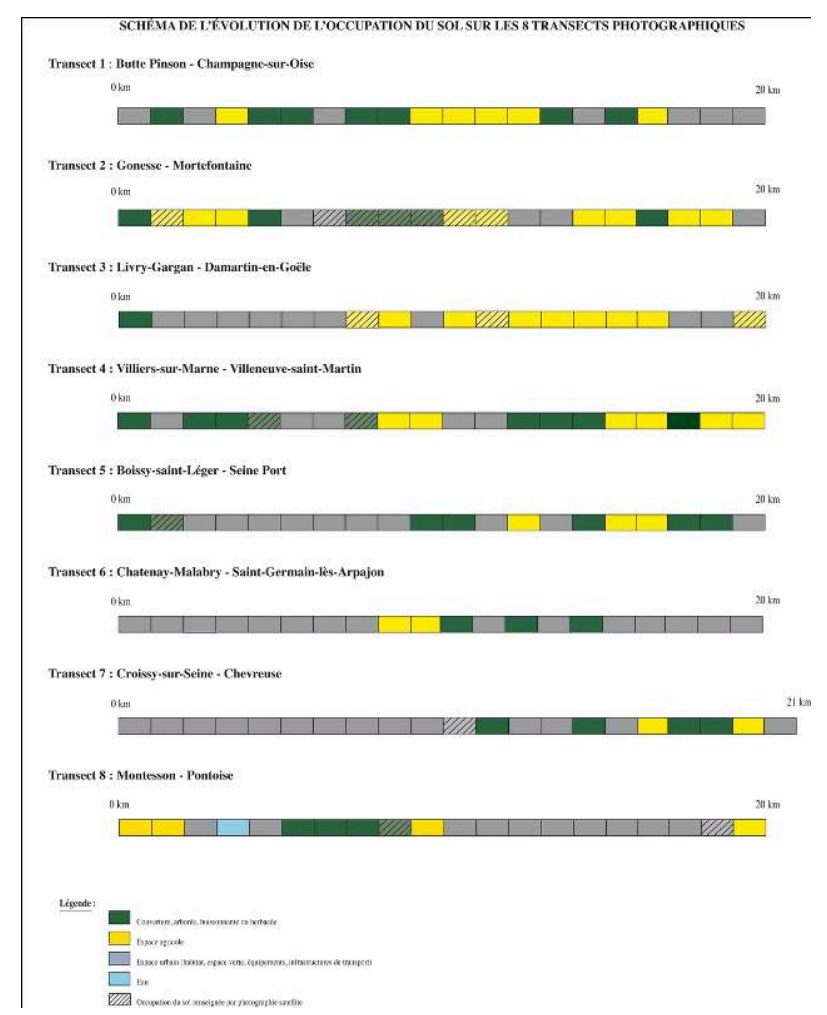

Une analyse plus approfondie montre que les arrangements paysagers identifiés sur chaque coupe paysagère sont complexes. Il est difficile de distinguer une logique d'homogénéité dans la transition de l'urbain vers le rural, même si l'on peut vérifier que, dans l'ensemble, le type d'occupation agricole se retrouve majoritairement dans la 
seconde moitié des transects, à plus de 10 kilomètres de la frange interne de la Ceinture verte. Certaines coupes vérifient globalement la logique du gradient centre-périphérie, comme le transect 5 , entre Boisssy-Saint-Léger et Seine-Port. Le transect 8 conduit entre Montesson et Pontoise fait figure ici d'exception puisque la coupe offre d'abord une occupation agricole, dans la plaine de Montesson avant qu'elle ne soit ensuite principalement urbaine, dans l'agglomération de Pontoise, cité médiévale enserrée dans l'urbanisation de la ville nouvelle de Cergy, à partir des années 1960. Le transect 6 entre Châtenay-Malabry et Saint-Germain-lès-Arpajon présente quant à lui une occupation urbaine largement dominante, ponctuée de quelques espaces végétalisés. L'espace y est densément urbanisé, avant de se faire plus étiré vers les bordures externes de la Ceinture verte. D'autres coupes paysagères, situées dans la partie nord de la Ceinture verte, en plaine de France, témoignent, eux, d'une occupation du sol largement agricole, entrecoupés par de grands axes de circulation routière. Enfin d'autres cas illustrent l'alternance entre les massifs forestiers et les espaces plus ruraux. Les forêts font ainsi office de tampon face à l'urbanisation.

La constitution d'une base de données photographique permet bien de renseigner sur les logiques paysagères à l'œuvre en Île-de-France de manière précise. On peut y voir des restructurations spatiales, comme l'émergence de polarités économiques secondaires ou des fronts d'urbanisation. Ces phénomènes se superposent à des logiques distinctes et parfois concomitantes en Ceinture verte: un gradient centrepériphérie et des logiques urbaines paysagères de vallée-plateau.

\section{Effets de ruptures et discontinuités paysagères en Ceinture verte}

18 La méthode des coupes paysagères révèle les nombreuses discontinuités, à la fois matérielles et symboliques. La réalisation des transects a ainsi été l'occasion d'expérimenter un parcours non linéaire et heurté. Vues au sol, ces discontinuités se manifestent sous forme de barrières concrètes, de murs et d'enclos, de coupures formées par les infrastructures de transports et des fermetures plus ou moins légales de l'espace. On repère aussi visuellement les effets de lisières et les zones de transitions entre deux paysages photographiés. L'impossibilité d'accéder à certains points lors de notre parcours, tout comme l'analyse des photographies recueillies, est un indice du rapport entre l'espace public et l'espace privé dans l'espace de Ceinture verte, à dominante périurbaine. La première situation rencontrée correspondant à un statut de propriété privée manifesté par des barrières physiques. Ainsi, enclos, murs, portails ou encore grillages ont rythmé notre itinéraire. Les transects, tracés en ligne droite, traversent des parcelles de jardins pavillonnaires, des grillages de locaux de grands groupes industriels de logistiques. Ce dernier cas a été rencontré le long des locaux de l'entreprise de transport FedEx à Roissy, où plusieurs points n'ont pas pu être photographiés, tout comme dans le périmètre d'une prison, enserrée dans une emprise de grande superficie et largement protégée à la maison d'arrêt du Val-d'Oise, à Osny.

Le deuxième élément empêchant l'accès aux points définis le long des tracés était celui d'une impossibilité due à des ruptures physiques des réseaux de transport difficilement franchissables à pied : routes et autoroutes fracturent le paysage. C'est le cas à MitryMory à proximité de l'aéroport de Roissy-Charles-de-Gaulle où le réseau routier, très dense, rend difficile l'accès aux terres agricoles, entièrement enclavées (illustration 3). Les infrastructures de communication, routières et ferroviaires, si elles permettent de 
relier les pôles urbains entre eux, constituent de véritables discontinuités à l'échelle locale.

Illustration 3 - Vue sur les champs et les D9 à Mitry-Mory

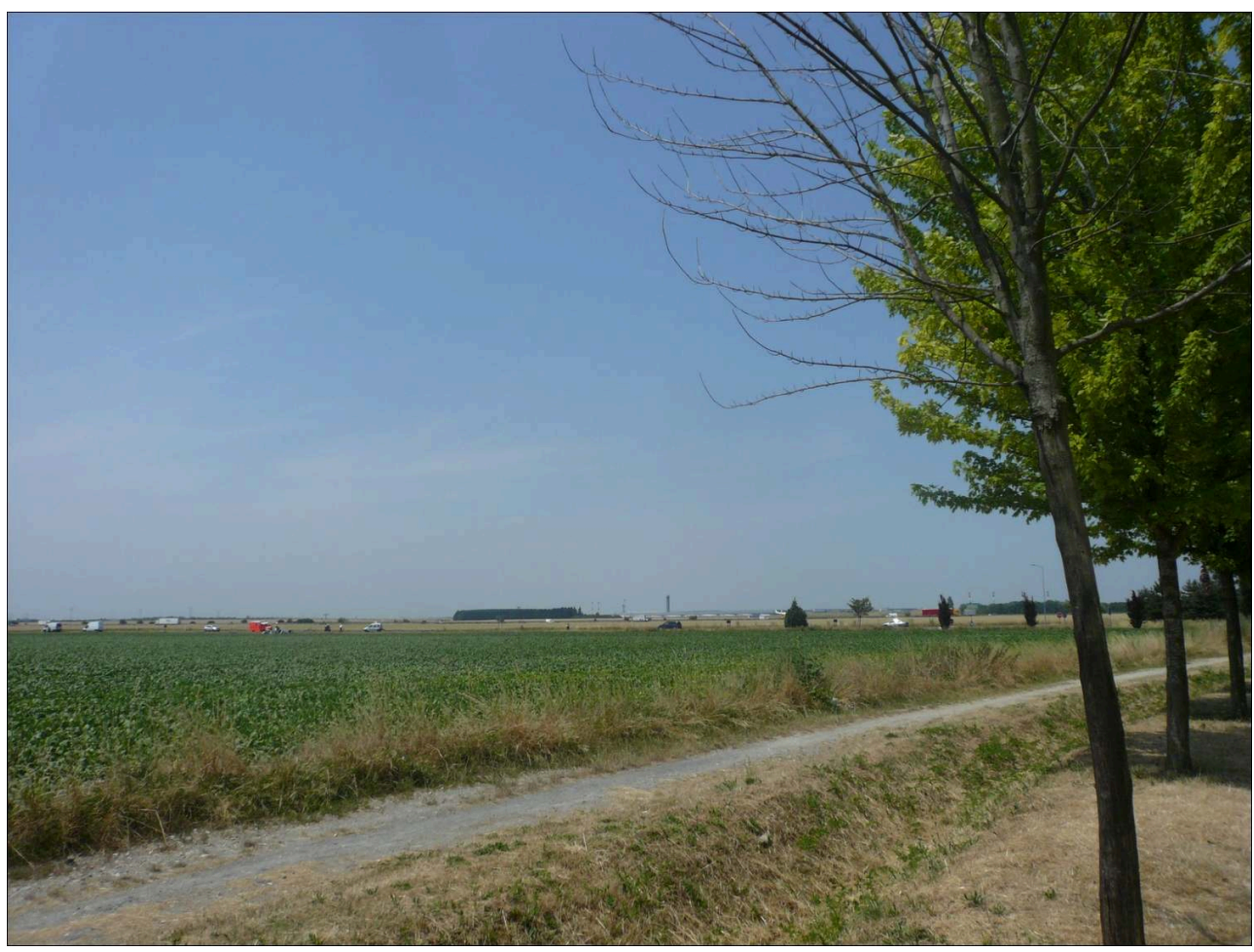

On distingue la tour de tour de contrôle de l'aéroport de Roissy-Charles-de-Gaulle. L'échangeur de I'A104 se situe un peu plus loin.

Auteur : M. Flégeau, juin 2014

En Ceinture verte, la densité de routes, d'autoroutes et de voies ferrées créée des ruptures physiques qui ne permettent pas aux habitants d'aller où bon leur semble. Il existe bien de nombreux ouvrages de franchissements dans les zones anciennement urbanisées, plus proche de l'aire urbaine centrale, mais lorsque l'urbanisation de l'espace a suivi la construction de ces infrastructures, la partition de l'espace reste importante. Par ailleurs, les lignes électriques à haute tension, si elles ne sont pas des coupures au sol, se manifestent également dans le paysage. Dans certains territoires, notamment dans le sud-est francilien, des pavillons sont établis sous les câbles des lignes électriques à haute tension. Ainsi, face à la pression de l'urbanisation, certains élus ont autorisé la construction de pavillons à ces emplacements, en non-conformité avec la règlementation en vigueur qui indique qu'une distance de 100 mètres doit être respectée entre les constructions et les lignes électriques aériennes à haute tension ${ }^{1}$. Ces dernières impriment d'autant plus l'aspect urbain de l'espace parcouru, lorsqu'elles traversent des paysages ouverts de plaines agricoles, où elles sont visibles de loin. 
Illustration 4 - Lignes électriques à haute tension au-dessus d'un rond-point à Champlan (91)

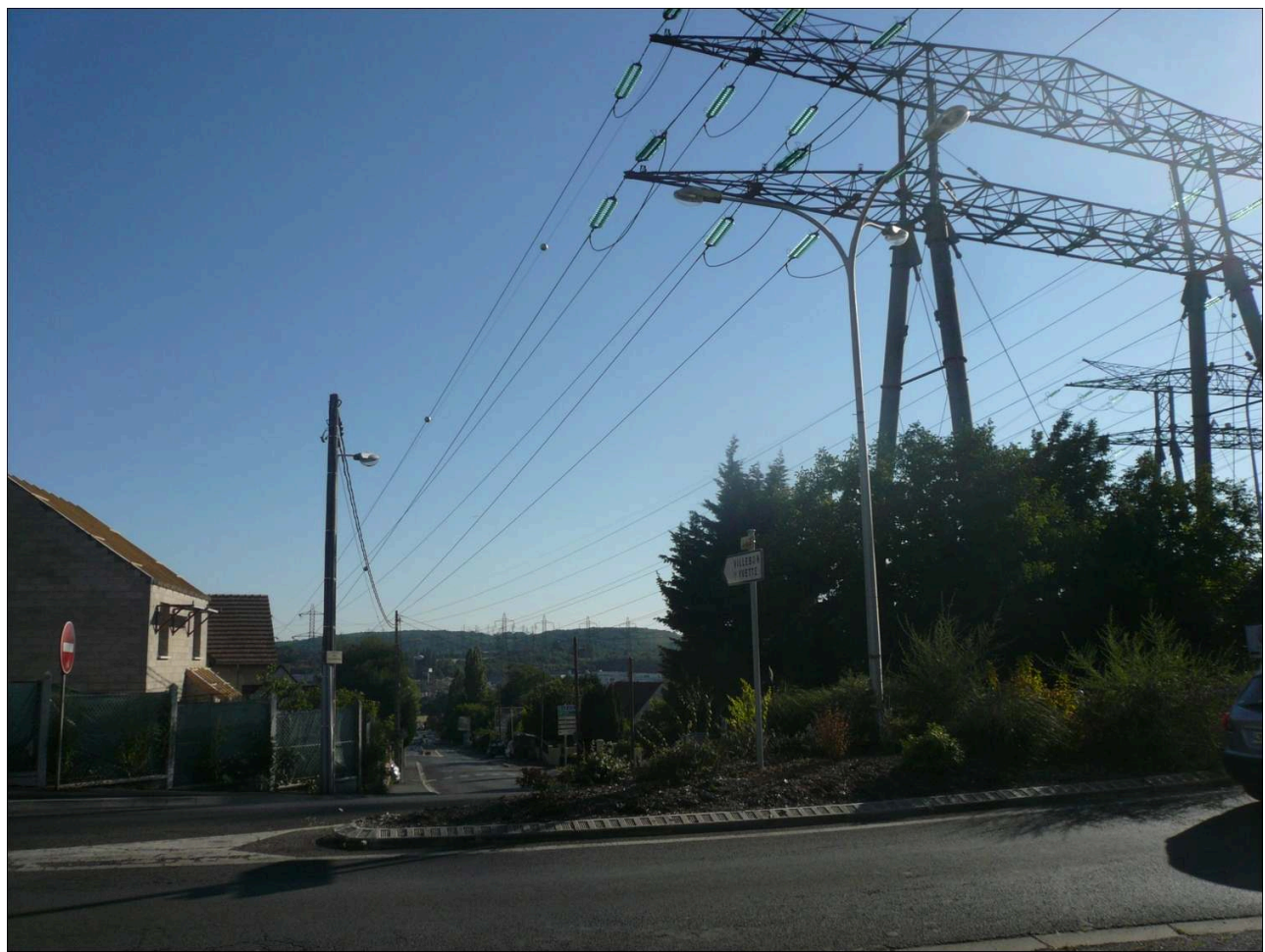

Les lignes se poursuivent vers la vallée de I'Yvette et le massif de la Bretèche.

Auteur : M. Flégeau, juin 2014

La méthode des transects photographiques constitue également un moyen d'observer les modalités d'appropriation de l'espace au travers de leur dimension visible dans le paysage, entre fermeture matérielle et immatérielle. La clôture matérielle marque l'appropriation de l'espace par un groupe social (Ripoll et Veschambres, 2005). Une très grande variété de ces barrières s'observe en Ceinture verte et l'on peut en dresser l'inventaire. Les clôtures enserrent tous types d'espaces, de l'habitat individuel aux zones industrielles et d'activités, en passant par des espaces non-bâtis. Dans ce dernier cas, la propriété privée, lorsqu'elle est matérialisée par des barrières, empêche l'accès aux espaces qu'urbanistes et paysagistes appellent pourtant des espaces ouverts (Banzo, 2015). De nombreux boisements privés sont ainsi enclos, tout comme les prairies et les pelouses appartenant au domaine privé. Le long de notre itinéraire, l'habitat individuel est pour l'essentiel clôturé. Le travail d'ethnologie conduit par P. Frileux (2010) sur les haies dans les franges pavillonnaires a montré l'importance de la mise en clôture de l'espace pour leurs habitants, dans un souci d'intimité et de protection. Si les CAUE (Conseils d'Architecture, d'Urbanisme et de l'Environnement) ont par exemple tenté d'harmoniser les paysages aux franges des villes en proposant des guides destinés aux propriétaires, c'est pourtant bien la diversité des clôtures qui se lit dans les paysages. Le PNR du Vexin édite ainsi des guides pour inciter les habitants à ne pas planter de haies monospécifiques avec des espèces comme le thuya ou le laurier du Caucase, largement utilisé dans le cas des lotissements pavillonnaire, qui peut aussi avoir un impact néfaste sur la biodiversité ${ }^{2}$, préoccupation récente des collectivités territoriales. Entre la haie végétale, les murs et murets de parpaing et les grilles en fer forgé de diverses hauteurs, ce besoin de protection de l'espace privé par les habitants participe à une fermeture de l'espace dans le périurbain, notamment en Ceinture verte. 
En France, la fermeture de l'habitat à l'échelle de la parcelle est une pratique courante, héritée de traditions culturelles et urbanistiques (Madoré, 2004). Un nombre très réduit d'ensembles résidentiels fermés, "clos, clôturé, fermé, protégé, sécurisé ou séparé " (Billard, Madoré, 2010) a été rencontré. L'accès à la résidence du "parc de Cerçay » à Villecresnes se fait par une route clôturée par une barrière. Les formes urbaines correspondant à l'habitat collectif - immeubles d'habitat collectif installés sur des bandes de pelouses -, sont ceints pour certains de barrières qui les séparent de l'espace public. La frontière entre l'espace privé et l'espace public est souvent repoussée à la rue.

Les barrières observées tout le long des transects sont pour l'essentiel concrètes et matérielles mais elles peuvent être aussi abstraites ou symboliques. La fermeture de l'espace est alors signifiée par des panneaux de signalétique. Ce signe, bien que matériel, suffit à manifester l'interdiction d'accès à l'espace. De la même manière qu'avec des clôtures, l'interdiction de pénétrer dans un espace pour l'ensemble des usagers est revendiquée par un groupe qui manifeste son usage exclusif de l'espace (Ripoll et Veschambres, 2005). Ces panneaux de signalisation témoignent d'un marquage de l'espace des propriétés privées grâce à des manifestations symboliques. Ces dernières sont donc particulièrement fortes. C'est le cas d'un boisement indiqué comme des zones de chasse à Émerainville, en limite du bois de Célie (illustration 5). Ici, les chasseurs ont délimité la zone de chasse réservée dont ils ont la jouissance exclusive par des panneaux d'interdiction d'entrer. À Pierrelaye, un panneau signifie également la propriété privée d'un bois, pour son usage exclusif.

Illustration 5 - Vue sur le chemin de Berdoudou à Émerainville

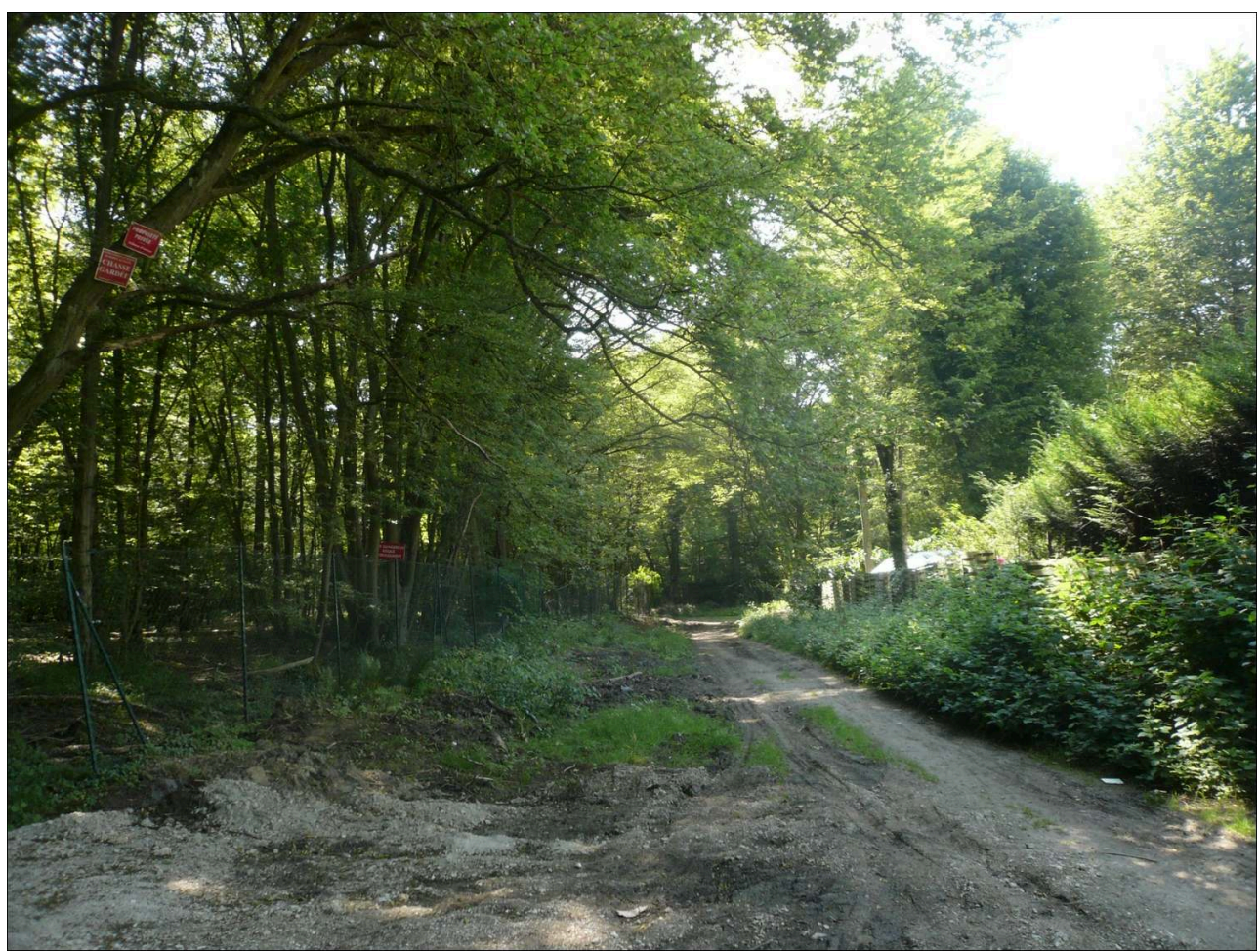

Aucune clôture ne délimite le boisement mais des panneaux signifiant une chasse gardée et une propriété privée en limite de la forêt régionale de Célie

Auteur: M. Flégeau, juin 2015. 
23 Ces barrières sont manifestes, sous forme d'enclos ou de panneaux indiquant la propriété privée du point de vue juridique. Pourtant, une appropriation illégale de l'espace peut toutefois aussi être affirmée par des enclosures. Ce type d'appropriation exclusive est fragile puisqu'il peut faire l'objet d'une éviction, notamment de la part des pouvoirs publics. La possibilité de l'appropriation illégale d'un espace est aussi ce qui justifie la mise en place de barrières. À Groslay, un enclos composé de bois et de ficelles a été installé dans une friche. Dans les documents d'urbanisme de la commune, cet espace n'est pas destiné à l'usage dont elle fait l'objet. Il est pourtant entretenu avec soin : l'herbe et coupée et les bordures des arbres fruitiers ont été nettoyés.

Illustratio 6 - Des barrières en bois délimitent un terrain de manière informelle dans une friche à Groslay

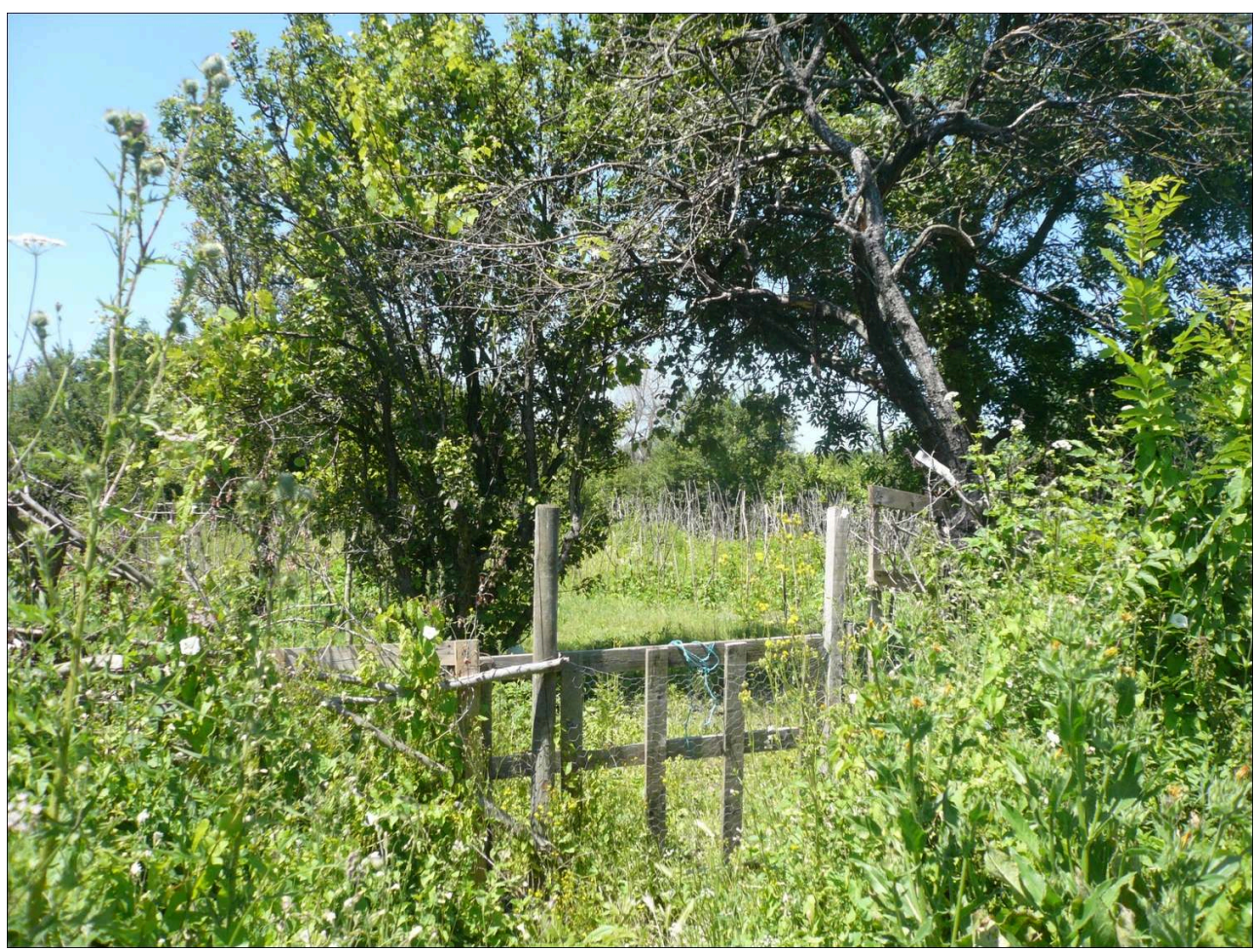

Auteur : M. Flégeau, juin 2014

Non loin de là, une autre friche, à Villetaneuse, a elle aussi été appropriée de manière informelle pour y installer un potager. Dans ce cas de figure, aucune marque de clôture de l'espace n'a été dessinée, l'appropriation n'étant pas nécessairement délimitée dans l'espace. La mise en place de barrières physiques permet en revanche une appropriation plus pérenne. 
Illustration 7 - Un potager informel installé dans une friche sur la commune de Villetaneuse

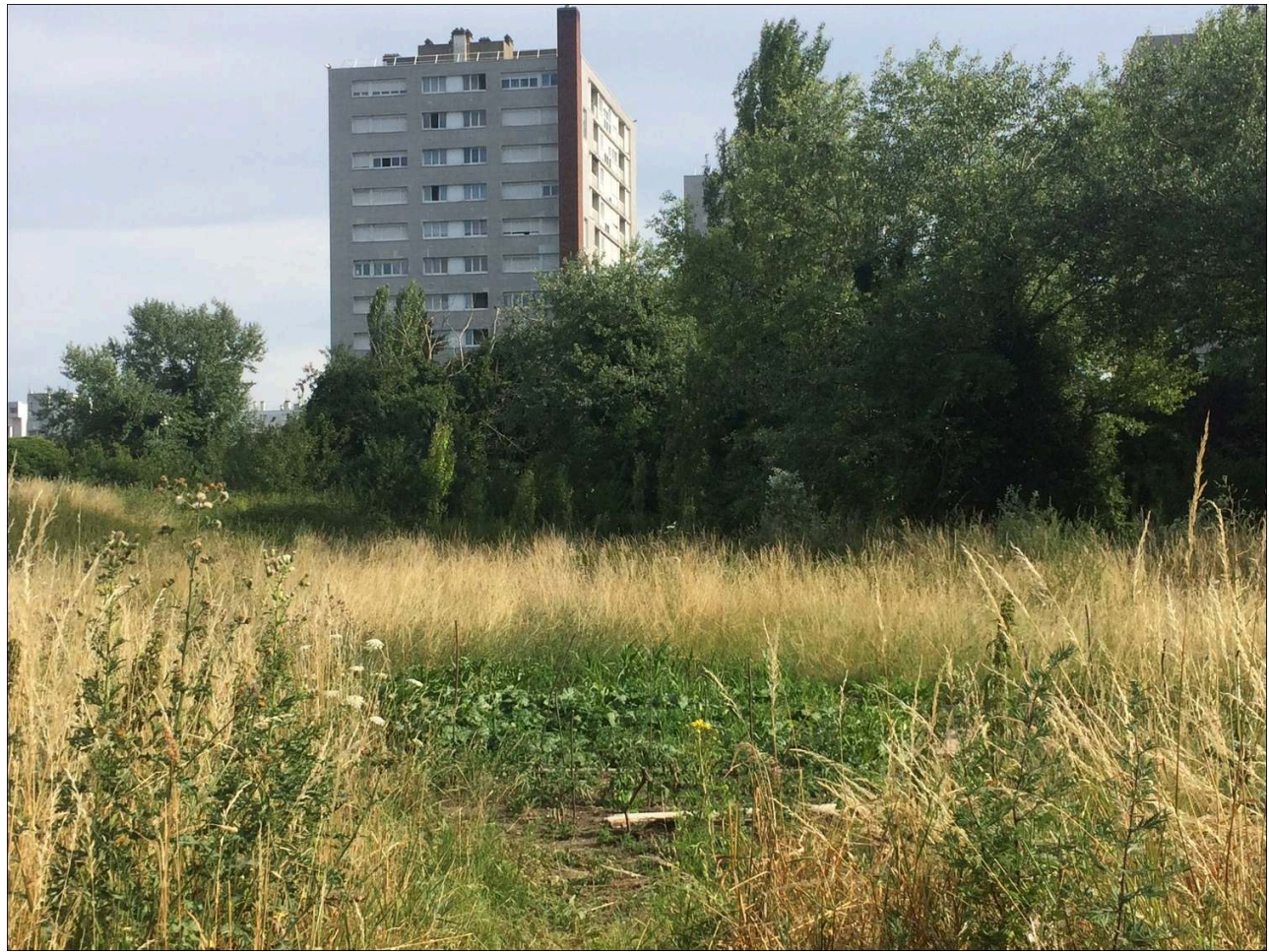

Auteur : M. Flégeau, juin 2015 s'observent, celles des aménagements à usage dissuasif. Les gestionnaires de l'aménagement local disposent des pierres imposantes pour empêcher l'installation de groupes qu'ils jugent indésirables. 
Illustration 8 : Des enrochements dans le parc régional de la Butte Pinson

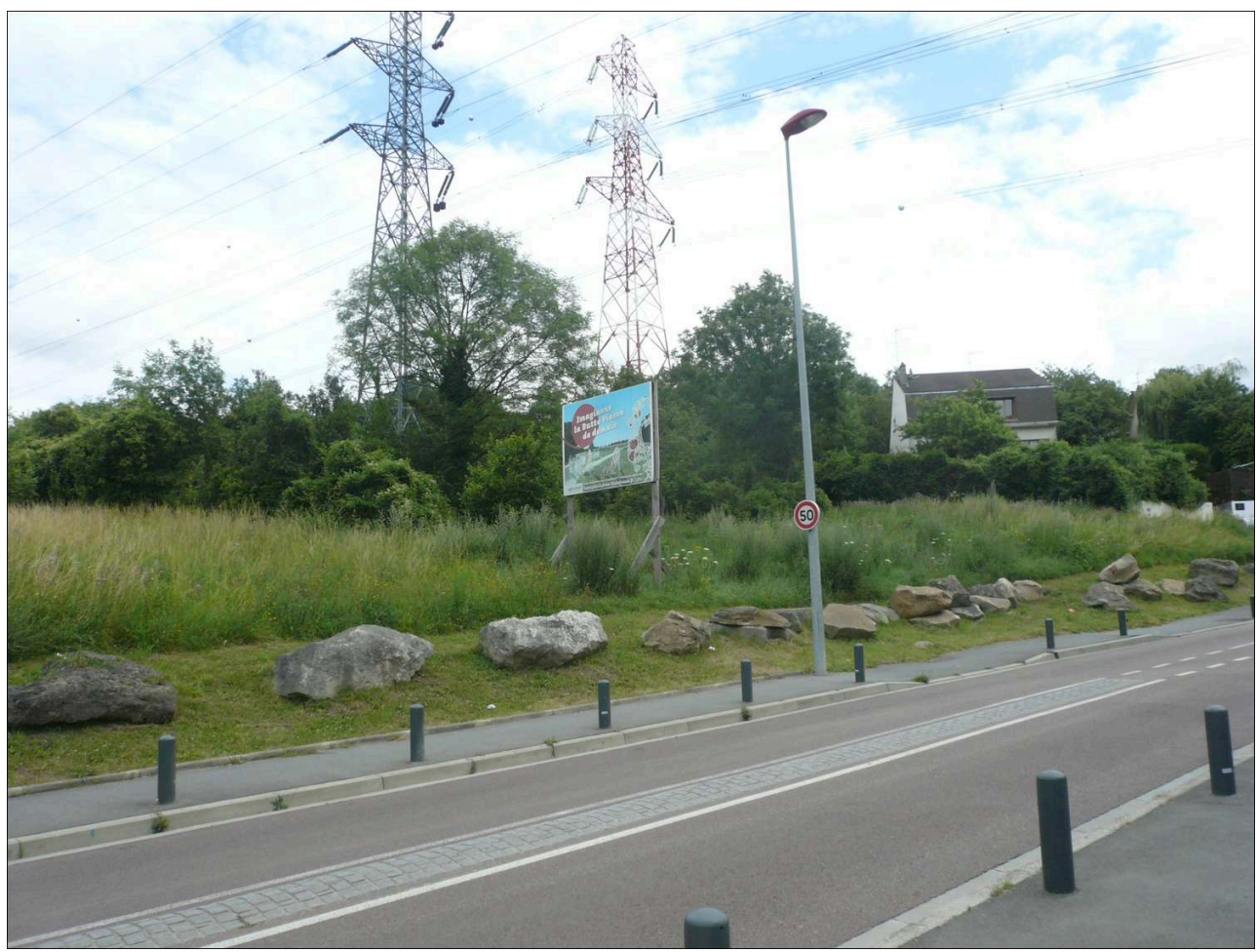

Auteur : M. Flégeau, juin 2015

Des enrochements ont été installés à la Butte Pinson pour éviter une appropriation de la partie du domaine temporairement en friche et destiné à devenir un parc régional. L'intercommunalité de Plaine Vallée confirme d'ailleurs l'utilisation de ces enrochements pour éviter la venue de gens du voyage. Ainsi, les gens du voyage voient leurs possibilités d'installation se restreindre dans l'ensemble de la Ceinture verte, espace ou les marges se ferment - souvent pour des motifs de mise en valeur paysagère (Flégeau, 2018b) - comme dans les parties centrales de l'agglomération. À Montesson ou encore à Ferrières, les enrochements servent à empêcher l'accès à des chemins agricoles ou à des routes d'accès à des bois privés. Ce sont souvent les espaces en attente de projet qui sont l'objet de ces dispositifs d'empêchement, ces derniers étant considérés comme temporaires.

\section{Zones de contacts, zones de transition}

Enfin, en grandissant l'échelle selon laquelle sont appréhendées les discontinuités, c'est-à-dire en passant de la cartographie à la photographie, les zones de transition peuvent également être pensées comme des ruptures, d'une épaisseur plus ou moins importante. Elles marquent ainsi le passage de l'urbain au rural. Il existe une multitude de zones de contact, qu'elles apparaissent entre zones pavillonnaires et espaces agricoles, comme à Savigny-le-Temple (illustration 9) ou entre zones industrielles et d'activités et boisements ou zones agricoles. Elles sont le plus souvent brutales, même si dans certains cas, on observe cependant des transitions plus douces. À Saint-Bricesous-Forêt, la transition entre les zones résidentielles et la forêt de Montmorency est occupée par une large bande de vergers plus ou moins en friche. Ce même constat peut d'ailleurs être fait tout le long de la forêt où les coteaux sont ponctuellement occupés 
par d'anciens vergers et des friches arbustives. La photographie permet de mettre en avant ces zones de contact, qui apparaissent sous forme de lisières pour l'observateur, enrichissant l'analyse cartographique des discontinuités spatiales.

Illustration 9 : Zone de contact entre un lotissement et des terres agricoles à Savigny-le-Temple

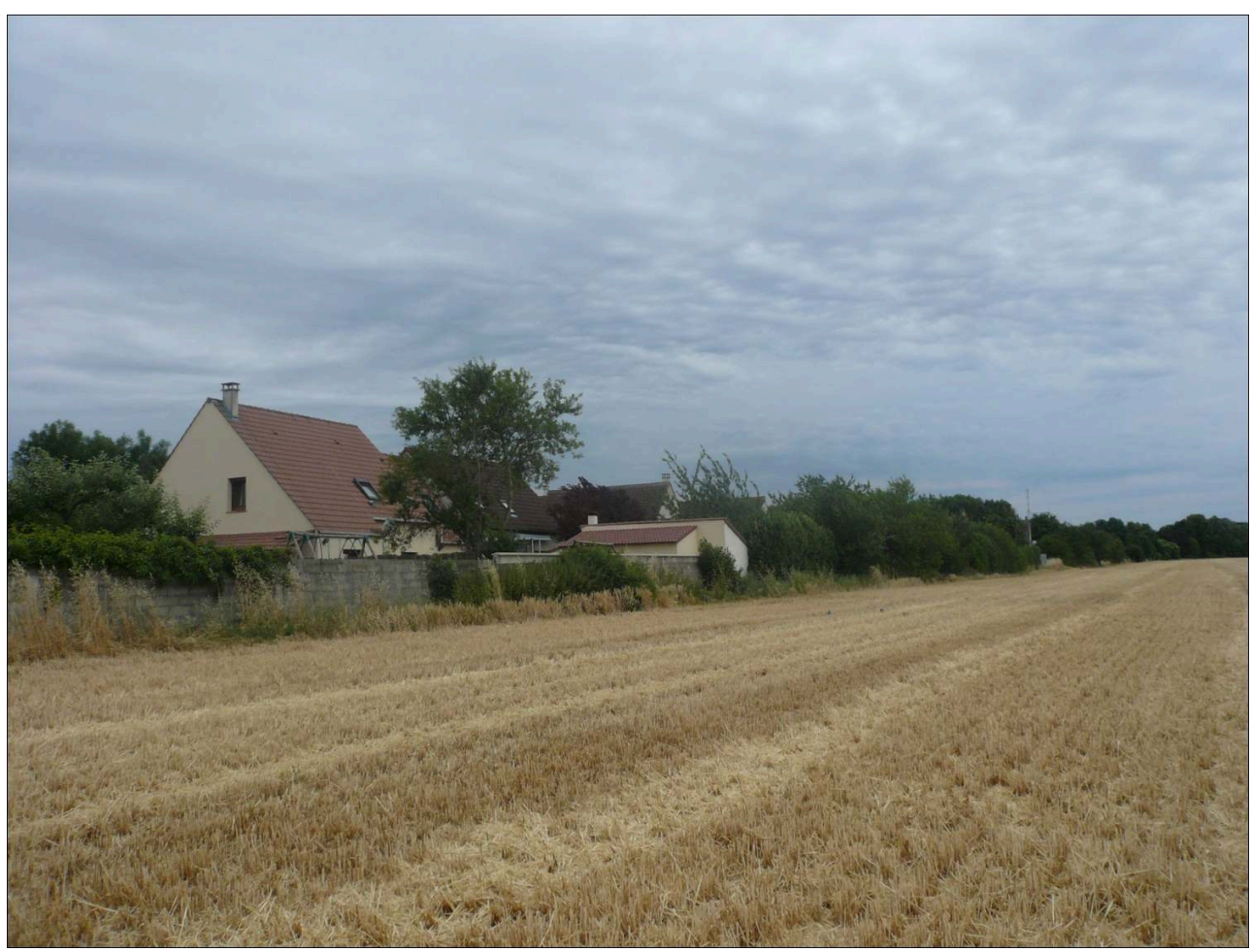

Auteur: M. Flégeau, juin 2014.

\section{Conclusion}

La réalisation des coupes paysagère a abouti à la constitution d'une base de données photographique permettant de documenter l'espace de Ceinture verte et ses caractéristiques. La méthode des transects photographiques a eu une place centrale dans notre démarche de recherche, placée en aval d'une approche cartographique, proposant une analyse globale, et en amont d'une enquête par entretiens pour appréhender les attentes environnementales des acteurs de l'aménagement et des habitants de la Ceinture verte. La mise en œuvre de cette méthode a été l'occasion de constater la grande fragmentation des paysages de Ceinture verte, déjà révélé par l'approche cartographique, par un travail au sol. Dans cet espace, largement hétérogène, se dessinent des logiques spatiales locales qui remettent en question l'idée d'un gradient d'urbanisation continu du centre de Paris vers ses marges. La méthode des transects photographiques apparaît donc comme un outil précieux pour appréhender la modulation des arrangements paysagers, en complément de la cartographie. La réalisation des coupes paysagères a aussi été l'occasion d'expérimenter directement les ruptures et les discontinuités dans le paysage lors d'un parcours personnel. L'espace de Ceinture verte est difficilement accessible dans un parcours radial à pied que nous avons réalisé. Cette expérience subjective renseigne ainsi sur les pratiques des habitants, notamment en ce qui concerne les espaces 
ouverts, s'ils ne sont pas dédiés à une ouverture au public. Par ailleurs, un jeu d'appropriation et de mise en clôture, matérielle ou symbolique, de l'espace y est aussi visible grâce à l'utilisation de la photographie. Qu'elle soit légale ou non, l'appropriation participe de la fermeture d'un espace largement périurbain. L'approche photographique peut donc être un outil intéressant pour amorcer une réflexion sur l'espace public et ses spécificités dans le périurbain.

La méthode des transects s'est voulue exploratoire. Des traitements statistiques de la base de données constituée ont aussi été envisagés et restent à poursuivre ${ }^{3}$. Au terme de ce parcours, il est utile de souligner quelques réflexions induites par l'approche du terrain expérimentée au travers de la photographie. Le passage du travail cartographique, soulignant des discontinuités et des ruptures à petite échelle, au travail photographique, conduit au sol, modifie la situation de l'observateur par rapport à son terrain. La traversée de l'espace de Ceinture verte par des transects photographiques implique la construction d'un regard spécifique, à la fois sur l'appréhension des dynamiques paysagères, l'expérience de l'accès aux espaces non bâtis mais aussi un rapport sensible à ces espaces et à ceux qui l'habitent, humains et aux autres qu'humains. Cette expérience du terrain qui a été la nôtre génère des effets sur la relation qu'entretient ensuite le chercheur avec les personnes enquêtées, une connaissance personnelle du terrain qu'il confronte au territoire vécu par l'habitant. Ces différents effets doivent bien sûr être pris en compte dans une réflexion globale sur le positionnement $d u$ chercheur par rapport à son terrain. L'articulation entre cartographie et photographie apporte une profondeur d'analyse pour appréhender les dynamiques spatiales dans un espace fortement hétérogène et transformé par des processus de planification ou d'urbanisation spontanée, et de renseigner sur la place qu'occupent les espaces non bâtis au sein de cet espace.

\section{BIBLIOGRAPHIE}

Alexandre F., 1994. Entre midis méditerranéen et atlantique, une transition phytoclimatique du Languedoc à l'Aquitaine. Thèse de doctorat de Géographie, Université Paris 7 - Denis Diderot.

Amati M. (dir.), 2008. Urban green belts in the twenty-first century. Aldershot, Hampshire, England ; Burlington, VT, Ashgate, $248 \mathrm{p}$.

Arrif T., Blanc N., Clergeau P., 2011. Trame verte urbaine, un rapport Nature - Urbain entre géographie et écologie. Cybergeo [En ligne]. URL: http://journals.openedition.org/cybergeo/24862

Banzo M., 2015. L'espace ouvert pour recomposer avec la matérialité de l'espace urbain. Articulo [En ligne], $\mathrm{n}^{\circ}$ 6. URL : http://journals.openedition.org/articulo/2708

Bigando E., 2013. De l'usage de la photo elicitation interview pour appréhender les paysages du quotidien : retour sur une méthode productrice d'une réflexivité habitante. Cybergeo [En ligne]. URL: http://journals.openedition.org/cybergeo/25919 
Billard G., Madoré F., 2010. Une géographie de la fermeture résidentielle en France. Quelle(s) méthode(s) de recensement pour quelle représentation du phénomène ? Annales de géographie, vol. $675, n^{\circ} 5$, p. $492-514$.

Bolon I., Cantoreggi N., Simos J., Ruiz de Castañeda R., 2019. Espaces verts et forêts en ville : bénéfices et risques pour la santé humaine selon l'approche «Une seule santé » (One Health). Santé Publique, vol. S1, n HS. p. 173-186.

Brunet R., François J.-C., Grasland C., 1997. La discontinuité en géographie : origines et problèmes de recherche. Entretien de Claude Graslan et Jean-Christophe François. L'Espace géographique [En ligne], vol. 26, nº 4, p. 297-308. URL: https://doi.org/10.3406/spgeo.1997.1096

Flégeau M., 2018a. La Ceinture verte d'île-de-France à l'épreuve du Grand Paris : effacement ou renouveau? Reconfigurations spatiales, territoriales et paysagères dans les marges de l'aire urbaine métropolitaine. Thèse de doctorat de Géographie et Aménagement, Université Paris 13 - SorbonneParis-Cité.

Flégeau M., 2018b. Populations reléguées et projets paysagers dans les marges de l'agglomération parisienne. Le cas de la plaine de Pierrelaye. EchoGéo [En ligne], n 46. UR : http:// journals.openedition.org/echogeo/16367

Frileux P., 2010. À l'abri de la haie dans le bocage pavillonnaire. Ethnologie française, vol. 4, $\mathrm{n}^{\circ} 4$, p. 639-648.

Gendrot C., 2002. L'investigation du territoire par l'image : apport de la notion de « série » dans l'exploitation de fonds photographiques. Espace géographique [En ligne], vol. 31, n 4, p. 357-368. URL: https://doi.org/10.3917/eg.314.0357

Laruelle N., Legenne C., 2004. La Ceinture verte d'île de France, un espace de vie à réinventer. Les Cahiers de l'IAU île-de-France, $\mathrm{n}^{\circ}$ 161, $44 \mathrm{p}$.

Lelli L., 2003. La photographie de paysage comme outil de mobilisation des acteurs pour un projet de territoire. In Debardieux B, Lardon S., Les figures du projet territorial. Éditions de l'Aube, La Tour-d'Aigues.

Lelli L., Paradis-Mandive, 2000. Quand le paysage ordinaire devient un paysage remarqué. SudOuest Européen, ${ }^{\circ}$ 7, p. 27-34.

Lizet B., de Ravignan F., 1994. Comprendre un paysage. Guide pratique de recherche. INRA, 147 p.

Luginbühl Y., 1989. Au-delà des clichés... La photographie du paysage au service de l'analyse. Strates. Matériaux pour la recherche en sciences sociales [En ligne], vol. 4. URL : http:// journals.openedition.org/strates/4072

Madoré F., 2004. Les ensembles résidentiels fermés en France : La forme d'habitat d'une société d'incertitude. Les Annales de la recherche urbaine, vol. 95, $\mathrm{n}^{\circ}$ 1, p. 98-106

Marshall A., 2009. La sensibilité photographique du géographe. EchoGéo [En ligne], nº 8. URL: http://journals.openedition.org/echogeo/11024

Métailié J.-P., 1995. Le photo-géographe et l’histoire des paysages. Histoire des séries, p. 91-95.

Meyer M., Papinot C., 2017. Le travail des images dans la démarche de recherche. Analyse réflexive et compréhension de l'objet. Images du travail, travail des images [En ligne], $\mathrm{n}^{\circ} 3$. URL: https://doi.org/10.4000/itti.1053

Poulot M., 2013. Du vert dans le périurbain. Les espaces ouverts, une hybridation de l'espace public (exemples franciliens). EspacesTemps.net [En ligne]. URL: https://www.espacestemps.net/ articles/du-vert-dans-le-periurbain/ 
Poulot M., Aragau C., Rougé L., 2016. Les espaces ouverts dans le périurbain ouest francilien : entre appropriations habitantes et constructions territoriales. Géographie, économie, société [En ligne], vol. 18, n 1. URL: http://ges.revuesonline.com/article.jsp?articleId=35949

Ripoll F., Veschambres V., 2005. Introduction. L'appropriation de l'espace : sur la dimension spatiale des inégalités sociales et des rapports de pouvoir. Norois [En ligne], vol. 2. URL: http:// journals.openedition.org/norois/477

\section{NOTES}

1. Arrêté du 17 mai 2001 fixant les conditions techniques auxquelles doivent satisfaire les distributions d'énergie électrique.

Voir

http://www.pnr-vexin-francais.fr/fichier/pnr_document/74/ document_fichier_fr_clotures.abords.pdf

3. L'importance de la base de données constituée nous a poussé à proposer l'ébauche d'un traitement statistique. Ce dernier a fait intervenir deux doctorants en mathématiques du Laboratoire de Traitement et Communication de l'Information, à Télécom ParisTech. Par la mise au point d'un algorithme, nous cherchions à décrypter les arrangements spatiaux qui révèleraient des logiques spatiales exposées. L'algorithme développé doit permettre de déceler des logiques de couleurs et de tenter d'identifier par différents critères photographiques (couleur, hauteur, taille, etc.) des types paysagers.

\section{RÉSUMÉS}

Cet article présente une méthode exploratoire mobilisant la photographie comme outil d'analyse des dynamiques paysagères dans un espace singulier, celui de la Ceinture verte d'île-de-France. L'usage de la photographie est pensé comme complémentaire d'une approche cartographique, conduite à l'échelle régionale. Des transects sont définis suivant des segments radiaux traversant l'épaisseur de la Ceinture verte. La base de données de clichés photographiques constituée permet d'analyser finement les logiques d'organisation paysagères, de se forger une expérience du terrain et d'amener une dimension sensible qui renseigne sur l'accès aux espaces non bâtis dans un espace à dominante périurbaine.

This article presents an exploratory method using photography as an analysis tool to understand landscape dynamics in the green belt of Paris Region. This method is considered as complementary to a mapping approach, developed at regional scale. Several transects are placed along radial segments across the green belt perimeter. The photographic database can be a way of analyzing landscape organization at local scale, to experience our study space and to bring a sensitive dimension, informing the access to the open spaces in a mostly peri-urban space. 
INDEX

Mots-clés : photographie, transect, paysage, discontinuité, usage

Keywords : photography, transect, landscape, discontinuity, use

Thèmes : Sur l'Image

\section{AUTEUR}

\section{MORGANE FLÉGEAU}

Morgane Flégeau , morgane.flegeau@gmail.com, est maître de conférence à l'Université de Lorraine, rattachée au LOTERR (Unité de recherche 7304). Elle a récemment publié :

- Flégeau M., Soubelet H., Carré S. et al., 2021. What are the possible effects of urban form on terrestrial biodiversity in western cities? A systematic map protocol. Environmental Evidence Journal [En ligne], vol. 10. URL: https://environmentalevidencejournal.biomedcentral.com/ articles/10.1186/s13750-021-00243-x - DOI: https://doi.org/10.1186/s13750-021-00243-x - Flégeau M., Roussel F., 2018. La ceinture verte de la Région Île-de-France : discontinuités, mises en valeur et représentations. In Rouget N., Schmitt G. (ed.), Nature des villes, nature des champs. Presses universitaires de Valenciennes, p. 145-168.

- Roussel F., Flégeau M., Lebeau B., Alexandre F., 2016. La plaine de Pierrelaye : un grand délaissé dans l'agglomération parisienne. In Grésillon E., Alexandre F., Sajaloli B. (ed.), La France des marges. Paris, Armand Colin, p. 306-301. 